\title{
Association between the effect of controlled fluid resuscitation on massive hemorrhage and expression of human neutrophil lipocalin
}

\author{
JIE-YANG YU, JIA-HUA PENG, LI HUI, HUI-QUAN HUANG, MING-HUA TAN and GUO JIAN \\ Intensive Care Unit, People's Hospital of Baise, Baise, Guangxi Zhuang Autonomous Region 533000, P.R. China
}

Received August 25, 2017; Accepted July 25, 2018

DOI: 10.3892/etm.2018.6591

\begin{abstract}
The present study was designed to investigate the association between the effect of controlled fluid resuscitation on massive hemorrhage and expression of human neutrophil lipocalin (HNL). A total of 112 patients confirmed with traumatic hemorrhage were enrolled as study subjects and were randomly divided into the control group $(\mathrm{n}=56)$ and observation group $(n=56)$. The control group was treated with rapid fluid resuscitation, and the observation group was treated with controlled fluid resuscitation. The success rate of resuscitation, incidence rate of complications, and HNL levels were compared both before and after resuscitation at multiple time intervals. The success rate of resuscitation showed a significant improvement while the incidence rate of complications were decreased. The HNL levels in both groups revealed increase after resuscitation at 3-10 h, thereby, they showed decline following peak point. However, the peak reduction in the observation group appeared earlier, while the HNL levels at 24 and $72 \mathrm{~h}$ were significantly lower than those in the control group. The study concluded that the effect of controlled fluid resuscitation on massive hemorrhage was superior to that of rapid fluid resuscitation. Moreover, controlled fluid resuscitation was also able to decrease the level of HNL as well as inflammatory response.
\end{abstract}

\section{Introduction}

Uncontrolled hemorrhagic shock (UHS) is a kind of clinically common disease, which is the 'lethal triad' with low body temperature, acidosis and coagulation dysfunction as typical pathophysiological changes. Further, its treatment is based primarily on the principle of fluid resuscitation that includes restoration of the blood perfusion and oxygen supply to end organs (1). The early fluid resuscitation program was based on

Correspondence to: Dr Jie-Yang Yu, Intensive Care Unit, People's Hospital of Baise, 8 Urban and Rural Road, Baise, Guangxi Zhuang Autonomous Region 533000, P.R. China

E-mail:sm0200402@163.com

Key words: controlled fluid resuscitation, neutrophil apolipoprotein, inflammatory response the Wiggers controlled hemorrhagic shock model (2). Earlier, the above method was utilized widely but a number of clinical observations revealing iatrogenic secondary strikes greatly affected its success rate (3). On the other hand, controlled fluid resuscitation emphasizes the maintenance of blood volume in basal metabolic cycle, instead of rapid stability of vital signs. In this way, it gave full play to the body's own compensatory mechanism and reduced the complications of fluid resuscitation (4). Furthermore, a study in the recent past has confirmed that stress as well as inflammatory response disorders played an important role in the development of UHS and during fluid resuscitation (5). A variety of inflammatory indexes, such as C-reactive protein (CRP), interleukin-6 (IL-6), tumor necrosis factor- $\alpha(\mathrm{TNF}-\alpha)$ and procalcitonin (PCT), have important application values in reflecting the severity of UHS as well as the effect of fluid resuscitation $(6,7)$. Moreover, recent studies have exhibited that human neutrophil lipocalin (HNL), is a kind of novel secreted protein in neutrophil secondary granules $(8,9)$. Furthermore, it was closely associated with inflammatory response, activation of signal transduction pathway, immune response and development of tumors. So, HNL has a better application value in diagnosing the early renal injury. Also, it helps to distinguish between acute, bacterial and viral infections $(10,11)$. Therefore, the present study aimed primarily to evaluate the association between the effect of controlled fluid resuscitation on massive hemorrhage as well as the HNL expression, which would contribute to the improvement of the assessment and treatment of UHS.

\section{Materials and methods}

Study design. A total of 112 patients diagnosed with UHS in People's Hospital of Baise from March 2015 to March 2017 were continuously selected as study subjects. Inclusion criteria were: i) patients with the trauma index $<12$ according to AIS90-ISS standard, injury severity score (ISS) $>16$ and survival time $>72 \mathrm{~h}$, accompanied with different degrees of shock (12); ii) patients who received effective treatment of traumatic disease; iii) patients who could undergo fluid resuscitation according to different groups, with complete clinical data and informed consent. Exclusion criteria were: i) patients complicated with serious underlying diseases, such as dysfunction in heart, liver, lung, kidney, brain or other organs, or coagulation dysfunction; ii) patients with uncontrolled infection or pain. The patients were divided into the 
control group $(n=56)$ and observation group $(n=56)$ using the random number method. The baseline data of the groups were comparable ( $\mathrm{P}>0.05)$ (Table I). The Ethics Committee of People's Hospital of Baise (Baise, China) duly approved the present study as well as all experimental methods. The consent forms were obtained from all the patients or their guardians before the treatment.

Research methods. The patients received immediate treatment in the Emergency Department or selective operation, followed by resuscitation and monitoring in ICU. They underwent general physical examination in accordance with 'CRASHPLAN' and 'VIPC' rescue process. At least two venous channels were established using the balanced crystalloid solution (lactated Ringer's solution or Ringer's sodium bicarbonate solution) supplemented by colloidal fluid (low molecular dextran and 706 dextran) at the right time with the crystal/colloid ratio of 2-3:1. According to the blood pressure recovery, the infusion rate, dose and vasoactive drugs used were determined. The control group was treated with rapid fluid resuscitation to maintain the mean arterial pressure (MAP) above $60 \mathrm{mmHg}$. The observation group was treated with controlled fluid resuscitation according to MAP or central venous pressure (CVP), and the MAP was maintained at approximately $50 \mathrm{mmHg}$ before hemostasis. After full hemostasis, MAP in both groups was maintained at $80-85 \mathrm{mmHg}$ and the hematocrit was maintained above $30 \%$. Moreover, the arterial oxygen saturation, blood routine, hepatic, renal and coagulation function as well as arterial blood gas, were monitored.

Observational indexes. The success rate of resuscitation, incidence rate of complications, and HNL levels at 3, 10, 24 and $72 \mathrm{~h}$ both before and after resuscitation were compared between the groups. Successful resuscitation was defined as follows: the bleeding as well as shock was controlled and consciousness was recovered. Moreover, blood pressure, heart rate, urine volume and CVP were returned to normal and were maintained for at least $24 \mathrm{~h}$ without obvious complications. The serum HNL level was detected via enzyme-linked immunosorbent assay (ELISA). The reagents were purchased from Jiangsu Beyotime Technology Co., Ltd. (Jiangsu, China). The level was measured three times strictly according to the instructions of kit and the average was recorded.

Statistical analysis. Statistical Product and Service Solutions (SPSS) 20.0 software (IBM Corp., Armonk, NY, USA) was utilized for the statistical analysis. Measurement data are presented as mean \pm standard deviation. The independent-samples t-test was used for comparison between the two groups, and the repeated measures analysis of variance and post hoc Tukey's test were performed and used for comparisons of data at different time-points. Enumeration data were presented as case or $\%$, and Chi-square test was used for the intergroup comparison. $\mathrm{P}<0.05$ was considered to indicate a statistically significant difference.

\section{Results}

Basic characteristics. The baseline data of the two groups are shown in Table I. There were no significant differences of
Table I. Comparison of baseline data between the two groups.

\begin{tabular}{lcccc}
\hline & $\begin{array}{c}\text { Control } \\
\text { group } \\
(\mathrm{n}=56)\end{array}$ & $\begin{array}{c}\text { Observation } \\
\text { group } \\
(\mathrm{n}=56)\end{array}$ & $\mathrm{t} / \chi^{2}$ & P-value \\
Items & $33 / 23$ & $35 / 21$ & 0.150 & 0.699 \\
\hline Male/female & $48.9 \pm 6.7$ & $47.6 \pm 6.5$ & 0.253 & 0.648 \\
Age (years) & $3.5 \pm 0.6$ & $3.7 \pm 0.9$ & 0.185 & 0.767 \\
Onset time (h) & $8.9 \pm 1.7$ & $8.6 \pm 1.5$ & 0.089 & 0.865 \\
Trauma index & $19.5 \pm 2.4$ & $21.2 \pm 2.5$ & 0.326 & 0.587 \\
ISS & & & 0.766 & 0.682 \\
Shock degree (n, \%) & $18(32.1)$ & $15(26.8)$ & & \\
Mild & $23(41.1)$ & $22(39.3)$ & & \\
Moderate & $15(26.8)$ & $19(33.9)$ & & \\
Severe & & & 0.301 & 0.960 \\
Trauma site (n, \%) & & & \\
Chest & $22(39.3)$ & $22(39.3)$ & & \\
Abdomen & $18(32.1)$ & $17(30.4)$ & & \\
Pelvic cavity & $6(10.7)$ & $5(8.9)$ & & \\
and head & & & & \\
Combined trauma & $10(17.9)$ & $12(21.4)$ & & \\
\hline
\end{tabular}

ISS, injury severity score.

gender ratio, age, onset time, trauma index, ISS, shock degree and trauma site between control group and observation group $(\mathrm{P}>0.05)$.

Comparison of success rate of resuscitation and incidence rate of complications. In the observation group, the success rates of resuscitation showed significant increase, while the incidence rate of complications showed significant decrease $(\mathrm{P}<0.05)$ (Table II).

Comparison of serum HNL levels at different time-points. The HNL levels in both groups were increased significantly reaching the peak at 3-10 $\mathrm{h}$ after resuscitation, and then were decreased. The HNL levels of the observation group at $10 \mathrm{~h}$ was similar to that at $3 \mathrm{~h}(\mathrm{P}>0.05)$. However, the HNL levels of the control group at $10 \mathrm{~h}$ was significantly higher than that at $3 \mathrm{~h}(\mathrm{P}<0.05)$. Moreover, the HNL levels were significantly decreased in both the control and observation groups at $24 \mathrm{~h}$ compared with that at $10 \mathrm{~h}(\mathrm{P}<0.05)$, which suggested that the time of peak reduction in the observation group appeared earlier (Fig. 1). Furthermore, the HNL levels of the observation group was significantly lower than that of the control group at 10, 24 and $72 \mathrm{~h}(\mathrm{P}<0.05)$ (Fig. 1).

\section{Discussion}

Wiggers controlled hemorrhagic shock model advocates that fluid resuscitation should be early and sufficient, while the fluid volume should be 2-3 times that of blood loss volume. At the same time, the positive inotropic drugs and vasoactive drugs should be used to raise the blood pressure, immediately. Furthermore, recovery and restoration of the effective blood 
Table II. Comparison of success rate of resuscitation and incidence rate of complications between the two groups (n, \%).

\begin{tabular}{|c|c|c|c|c|c|c|c|}
\hline Groups & $\mathrm{n}$ & Success rate & $\begin{array}{c}\text { Dilutional } \\
\text { coagulation } \\
\text { disorders }\end{array}$ & $\begin{array}{c}\text { Detachment of } \\
\text { thrombus }\end{array}$ & Rebleeding & $\begin{array}{l}\text { Pulmonary edema or } \\
\text { respiratory distress } \\
\text { syndrome }\end{array}$ & Complications \\
\hline Control group & 56 & $44(78.6)$ & $3(5.4)$ & $2(3.6)$ & $4(7.1)$ & $3(5.4)$ & $12(21.4)$ \\
\hline Observation group & 56 & $52(92.9)$ & 0 & $1(1.8)$ & $2(3.6)$ & $1(1.8)$ & $4(7.1)$ \\
\hline$\chi^{2}$ & & 4.667 & & & & & 4.667 \\
\hline P-value & & 0.031 & & & & & 0.031 \\
\hline
\end{tabular}

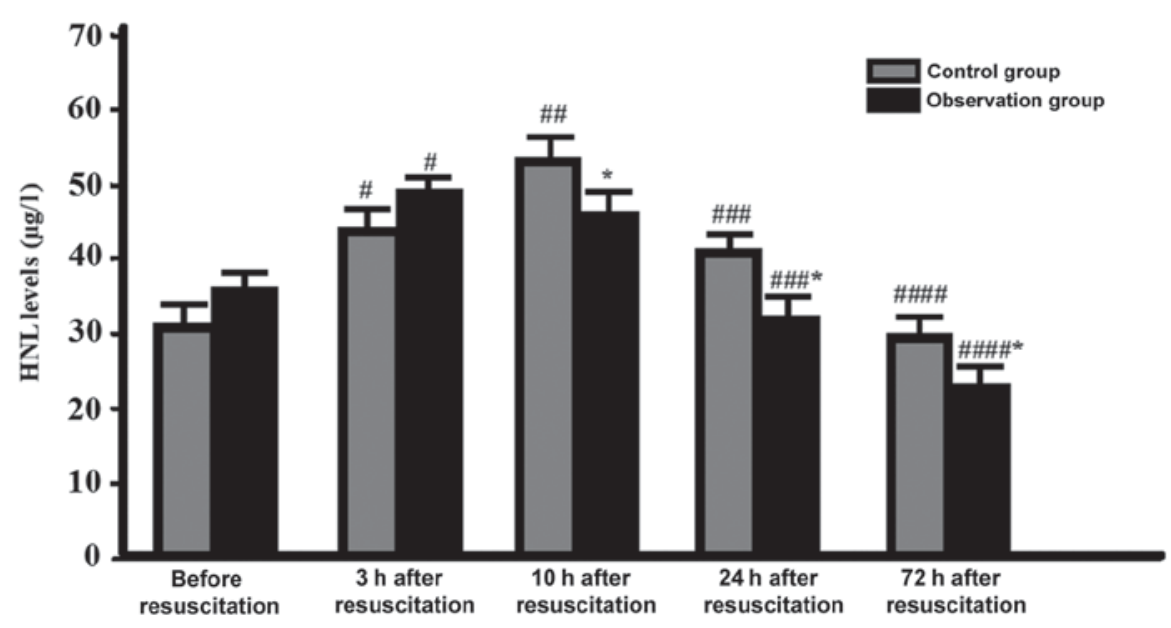

Figure 1. Detection of serum HNL levels in the two groups at different time-points before and after resuscitation via ELISA ( ${ }^{\mathrm{P}<0.05}$, comparison of serum HNL levels in the two groups before and at $3 \mathrm{~h}$ after resuscitation; ${ }^{\# \#} \mathrm{P}<0.05$, comparison of serum HNL levels in the control group at 3 and $10 \mathrm{~h}$ after resuscitation; ${ }^{\# \#} \mathrm{P}<0.05$, comparison of serum HNL levels in the two groups at 10 and $24 \mathrm{~h}$ after resuscitation; ${ }^{\# \# \#} \mathrm{P}<0.05$, comparison of serum HNL levels in the two groups at 24 and $72 \mathrm{~h}$ after resuscitation; ${ }^{*} \mathrm{P}<0.05$, comparisons of serum HNL levels between the observation and control groups at 10,24 and $72 \mathrm{~h}$ after resuscitation). HNL, human neutrophil lipocalin.

volume should be performed as soon as possible. This ensures effective perfusion of organs as well as tissues. Also, the traumatic hemorrhagic shock is closely related to the mortality rate of patients and incidence rate of complications (13). However, before the active bleeding could be effectively controlled, a large amount of fluid resuscitation would result in the rise of blood loss leading to the dilution of coagulation dysfunction (14). Also, a recent report observed significant damage to the mitochondrial function along with the reduction in the oxygen supply to tissues causing acidosis (15). At the same time, a large amount of rapid infusion has been reported to cause decrease in the vasoconstriction, leading to thrombus translocation, increased complications and high mortality rates (16). The concept of controlled fluid resuscitation is to reduce the harmful effects of early massive fluid resuscitation, effectively so as to improve the perfusion as well as oxygen supply to organ tissues in shock phase. This in turn reduced the occurrence of traumatic coagulopathy without causing over-diluted blood. It also effectively prevented the detachment of thrombus as well as bleeding within the allowable low blood pressure range. Furthermore, it caused reduction in apoptosis while maintaining the compensatory mechanism thereby, improving the survival rate (17). In addition, the type and dosage of resuscitation fluid and monitoring of the resuscitation effects are also important contents of recovery management. In addition to the traditional blood pressure, heart rate, urine volume and CVP (as the parameters of full perfusion and resuscitation outcomes), the non-invasive cardiac output, cardiac ultrasound, pulse indicator continuous cardiac output monitoring, blood lactate, base deficit, intestinal mucosal $\mathrm{pH}$, central venous oxygen saturation, orthogonal polarization spectral imaging and bypass dark-field imaging technique, have a certain application value in accurate assessment of the resuscitation time and mode $(18,19)$. At the same time, selection of biochemical markers with higher sensitivity as well as specificity to assess the resuscitation effect is also an important task of UHS.

HNL, also known as neutrophil gelatinase associated lipocalin, belongs to the lipocalin superfamily that exists mainly in the form of monomer (25 kD), homodimer (45 kD) and heteromultimer covalently bound to neutrophil gelatinase. HNL is a kind of iron carrier protein, whose main ligand is a small iron-binding molecule. When the neutrophils are activated or epithelial cells are damaged under inflammatory stimulation, HNL is often expressed on a large scale (20). The present study showed that the serum HNL was at a high level before UHS resuscitation, indicating that HNL was expressed in the early stage of UHS and was involved in the occurrence as well as development of the disease. HNL, as an important molecular marker in the early inflammatory response of the body, is the product secreted by neutrophil activation. It is the chemotactic factor of a variety of inflammatory cells and the 
inducible factor of a variety of inflammatory mediators that are core molecule in the inflammatory cascade reaction (21). The serum HNL levels in both groups were further increased after resuscitation at $3 \mathrm{~h}$, and it reached the peak in the observation group. However, it was not significantly different when compared with the control group. This confirmed that the fluid resuscitation may further activate the inflammatory response in the body and is possibly the result of further development of UHS. In the control group, the serum HNL level was increased to the peak at $10 \mathrm{~h}$ after resuscitation, indicating that the early rapid fluid resuscitation may also be responsible for observed increase of the degree of inflammatory response in the body. The above observation could be due to the massive rapid fluid resuscitation, leading to an inflammatory response in the body. The serum HNL levels in both groups began to decrease gradually at $24 \mathrm{~h}$ after resuscitation, and the level in the observation group was significantly lower than that in the control group. This suggested that HNL has a certain application value in the early evaluation of inflammatory response degree and has confirmed effects of fluid resuscitation on UHS.

There were also some limitations in this study. The sample size is relatively small and the follow-up time is relatively short. The signal pathway was not investigated in this study. Further study with a lager sample size and longer follow-up time or focused on the mechanism is still needed.

In conclusion, hemorrhagic shock produces hemodynamic changes that often result in a systemic inflammatory response that can lead to multiple organ failure and death. Early recognition of hemorrhagic shock is paramount in civilian and military communities to effectively implement damage-control resuscitation strategies to prevent progression into a state of irreversible shock and permit appropriate allocation of resources. The present study concludes that the controlled fluid resuscitation on massive hemorrhage is superior to that of rapid fluid resuscitation. Also, controlled fluid resuscitation could decrease the HNL levels and inflammatory response. Our results may promote the improvement of the assessment and treatment of UHS.

\section{Acknowledgements}

Not applicable.

\section{Funding}

No funding was received.

\section{Availability of data and materials}

The datasets used and analyzed during the current study are available from the corresponding author on reasonable request.

\section{Authors' contributions}

JYY wrote the paper, analyzed the data, conceived and designed the experiments. JYY, JHP and LH performed the experiments. HQH, MHT and GJ contributed reagents/materials/analysis tools. All authors read and approved the final manuscript.

\section{Ethics approval and consent to participate}

The Ethics Committee of People's Hospital of Baise (Baise, China) approved the present study. The consent forms were obtained from all the patients or their guardians.

\section{Patient consent for publication}

Not applicable.

\section{Competing interests}

The authors declare that they have no competing interests.

\section{References}

1. Zhao G, Wu W, Feng QM and Sun J: Evaluation of the clinical effect of small-volume resuscitation on uncontrolled hemorrhagic shock in emergency. Ther Clin Risk Manag 13: 387-392, 2017.

2. Li T, Zhu Y, Tian K, Xue M, Peng X, Lan D and Liu L: Ideal resuscitation pressure for uncontrolled hemorrhagic shock in different ages and sexes of rats. Crit Care 17: R194, 2013.

3. Ertmer C, Kampmeier T, Rehberg S and Lange M: Fluid resuscitation in multiple trauma patients. Curr Opin Anaesthesiol 24: 202-208, 2011.

4. Duan C, Li T and Liu L: Efficacy of limited fluid resuscitation in patients with hemorrhagic shock: A meta-analysis. Int J Clin Exp Med 8: 11645-11656, 2015.

5. Moran A, Thacker SA, Arikan AA, Mastrangelo M-AA, Wu Y, Yu B and Tweardy DJ: IL-6-mediated activation of Stat $3 \alpha$ prevents trauma/hemorrhagic shock-induced liver inflammation. PLoS One 6: e21449, 2011.

6. Liappis AP, Gibbs KW, Nylen ES, Yoon B, Snider RH, Gao B and Becker KL: Exogenous procalcitonin evokes a pro-inflammatory cytokine response. Inflamm Res 60: 203-207, 2011.

7. Wright EH and Khan U: Serum complement-reactive protein (CRP) trends following local and free-tissue reconstructions for traumatic injuries or chronic wounds of the lower limb. J Plast Reconstr Aesthet Surg 63: 1519-1522, 2010.

8. Constantin JM, Futier E, Perbet S, Roszyk L, Lautrette A Gillart T, Guerin R, Jabaudon M, Souweine B, Bazin J-E, et al: Plasma neutrophil gelatinase-associated lipocalin is an early marker of acute kidney injury in adult critically ill patients: A prospective study. Crit Care 25: 176.e10-176.e6, 2010.

9. Cruz DN, de Cal M, Garzotto F, Perazella MA, Lentini P Corradi V, Piccinni P and Ronco C: Plasma neutrophil gelatinase-associated lipocalin is an early biomarker for acute kidney injury in an adult ICU population. Intensive Care Med 36: 444-451, 2010.

10. Portal AJ, McPhail MJ, Bruce M, Coltart I, Slack A, Sherwood R, Heaton ND, Shawcross D, Wendon JA and Heneghan MA: Neutrophil gelatinase - associated lipocalin predicts acute kidney injury in patients undergoing liver transplantation. Liver Transpl 16: 1257-1266, 2010.

11. de Geus HR, Bakker J, Lesaffre EM and le Noble JL: Neutrophil gelatinase-associated lipocalin at ICU admission predicts for acute kidney injury in adult patients. Am J Respir Crit Care Med 183: 907-914, 2011.

12. Baker SP, O'Neill B, Haddon W Jr and Long WB: The injury severity score: A method for describing patients with multiple injuries and evaluating emergency care. J Trauma 14: 187-196, 1974.

13. Morrison CA, Carrick MM, Norman MA, Scott BG, Welsh FJ, Tsai P, Liscum KR, Wall MJ Jr and Mattox KL: Hypotensive resuscitation strategy reduces transfusion requirements and severe postoperative coagulopathy in trauma patients with hemorrhagic shock: Preliminary results of a randomized controlled trial. J Trauma 70: 652-663, 2011.

14. White NJ, Wang X, Liles C and Stern S: Fibrinogen concentrate improves survival during limited resuscitation of uncontrolled hemorrhagic shock in a Swine model. Shock 42: 456-463, 2014.

15. Li T, Zhu Y, Hu Y, Li L, Diao Y, Tang J and Liu L: Ideal permissive hypotension to resuscitate uncontrolled hemorrhagic shock and the tolerance time in rats. Anesthesiology 114: 111-119, 2011. 
16. Rezende-Neto JB, Rizoli SB, Andrade MV, Lisboa TA and Cunha-Melo JR: Rabbit model of uncontrolled hemorrhagic shock and hypotensive resuscitation. Braz J Med Biol Res 43: $1153-1159,2010$.

17. Lu YQ, Cai XJ, Gu LH, Fan YJ, Wang Q and Bao DG: Effects of three fluid resuscitation methods on apoptosis of visceral organs in rats with hemorrhagic shock. J Zhejiang Univ Sci B 6: 907-912,2005

18. Ho L, Lau L, Churilov L, Riedel B, McNicol L, Hahn RG and Weinberg L: Comparative evaluation of crystalloid resuscitation rate in a human model of compensated haemorrhagic shock. Shock 46: 149-157, 2016.

19. De Backer D, Ospina-Tascon G, Salgado D, Favory R, Creteur J and Vincent JL: Monitoring the microcirculation in the critically ill patient: Current methods and future approaches. Intensive Care Med 36: 1813-1825, 2010.
20. Venge P, Eriksson A-K, Douhan-Håkansson L and Pauksen K: Human neutrophil lipocalin in activated whole blood is a specific and rapid diagnostic biomarker of bacterial infections in the respiratory tract. Clin Vaccine Immunol 24: e00064-e17, 2017.

21. Venge P, Douhan-Håkansson L, Garwicz D, Peterson C, Xu S and Pauksen K: Human neutrophil lipocalin as a superior diagnostic means to distinguish between acute bacterial and viral infections. Clin Vaccine Immunol 22: 1025-1032, 2015.

This work is licensed under a Creative Commons

Attribution-NonCommercial-NoDerivatives 4.0 International (CC BY-NC-ND 4.0) License. 